\title{
Heterodyne Doppler global velocimetry
}

\section{Conference Paper}

Author(s):

Meier, Alexander H.; Rösgen, Thomas

Publication date:

2009-10

Permanent link:

https://doi.org/10.3929/ethz-b-000058940

Rights / license:

In Copyright - Non-Commercial Use Permitted

Originally published in:

Experiments in Fluids 47(4-5), https://doi.org/10.1007/s00348-009-0647-0 


\title{
Heterodyne Doppler global velocimetry
}

\author{
Alexander H. Meier · Thomas Roesgen
}

Received: 30 September 2008/Revised: 3 March 2009/Accepted: 12 March 2009/Published online: 29 March 2009

(C) Springer-Verlag 2009

\begin{abstract}
Doppler Global Velocimetry (DGV) is an imaging flow measurement technique which allows the measurement of the velocity distribution in a plane. In DGV the frequency shift of scattered light from moving particles within the flow is used to determine the local flow velocity. Heterodyne Doppler Global Velocimetry (HDGV) is a new approach which combines the imaging and geometrical characteristics of DGV with the measurement principles of reference beam laser Doppler anemometry. The frequency shifted scattered light from the flow tracers is heterodyned with a reference beam from the same light source. Due to interference the result of this superposition is a harmonic intensity modulated signal. This signal is detected using a smart pixel detector array to obtain the velocity distribution. Two different experiments are presented. The first experiment compares the measured velocity distribution of a rotating disk with its actual velocity. The second experiment demonstrates the capability of the technique to measure a real flow.
\end{abstract}

\section{Introduction}

The measurement of flow velocity distributions based on optical Doppler shift detection remains an active field of research in flow diagnostics. By measuring the frequency shift of light scattered off moving particles, Doppler

\footnotetext{
A. H. Meier $(\bowtie) \cdot T$. Roesgen

Institute of Fluid Dynamics, ETH Zurich,

Sonneggstrasse 3, 8092 Zurich, Switzerland

e-mail: meier@ifd.mavt.ethz.ch

T. Roesgen

e-mail: roesgen@ifd.mavt.ethz.ch
}

Global Velocimetry (DGV) avoids some of the restrictions of Particle Image Velocimetry (PIV). Without the need for resolving individual particles, the method may be particularly well suited for large-scale applications. Standard DGV techniques rely on the use of atomic line filters to convert the frequency shift into a change in intensity (Ainsworth et al. 1997; Elliott et al. 1999; Samimy et al. 2000). The technique has been successfully applied in full scale wind tunnels (Beutner et al. 1998), high-speed flows (Smith et al. 1996) and combustion measurements (Roehle et al. 2000). We propose a new approach to measure global Doppler frequency shifts. In Heterodyne Doppler Global Velocimetry (HDGV) the frequency shifted light scattered from the flow tracers is demodulated with a reference beam split off from the same coherent light source. Due to interference the result of this superposition is a harmonic intensity modulated signal. The frequency of the modulation represents the Doppler shift of the scattered light. This frequency is in the order of $1 \mathrm{Mhz} /(\mathrm{m} / \mathrm{s})$ which is too high to be sensed directly using conventional integrating camera technologies. Instead, a smart pixel imaging array is used which is capable of performing coherent "lock-in" detection for frequencies up to $50 \mathrm{kHz}$ (Beer et al. 2005). The demodulated image data is further processed using an algorithm based on pulse pair statistics (Miller et al. 1972) to obtain the velocity distribution. This latter technique is attractive in that it can detect and analyze a complete range of frequencies in a single image record and does not require a systematic frequency sweep. The maximum demodulation frequency of the available detector limits the measurement range to velocities up to $0.1 \mathrm{~m} / \mathrm{s}$. This is quite different from conventional DGV which usually deals with Doppler shifts in the order of several tens of Mhz and uncertainties in the order of a few Mhz (Elliott et al. 1999). 


\section{Method}

This section consists of two parts. The first subsection presents a simple model of the signal formation for HDGV which consists of the basics of DGV and the mixing process of HDGV. The second subsection describes the special detector array used to detect the signal, its pixel-level signal processing and the subsequent post-processing to derive the velocity distribution.

\subsection{Signal formation}

The frequency $v$ of the light scattered off an illuminated moving particle is shifted according to Eq. 1.

$v-v_{0}=v_{\mathrm{d}}=\frac{1}{\lambda_{0}}(\mathbf{O}-\mathbf{I}) \cdot \mathbf{V}$

The Doppler shift $v_{\mathrm{d}}$ depends on the velocity and direction of motion $\mathbf{V}$ of the scattering particle, the wavelength $\lambda_{0}$, the direction I of the illumination and the direction of observation $\mathbf{O}$. According to Eq. 1 the measurement of $v_{\mathrm{d}}$ allows the reconstruction of one specific velocity component, namely the projection $\mathbf{V}$ of onto the bisecting line of the two directions $\mathbf{I}$ and $\mathbf{O}$. Figure 1 shows the geometry of a DGV set up indicating the arrangement of the vectors in Eq. 1.

In HDGV the frequency shifted light from the particles is mixed on the detector surface with a reference light wave which stems from the same source. If the path length difference between the light from the particles and the reference light wave is within the coherence length of the light source they interfere with each other. The observable signal $S$ of this mixing process for a single scattering particle becomes:

$S=A_{\mathrm{Sig}}^{2}+A_{\mathrm{Ref}}^{2}+2 A_{\mathrm{Sig}} A_{\mathrm{Ref}} \cos \left(2 \pi v_{\mathrm{d}} t+\phi\right)$

where $A_{\text {Ref }}$ denotes the intensity of the reference wave, $A_{\mathrm{Sig}}$ the intensity of the signal wave and $\phi$ is a random phase shift which is assumed to be constant during the scattering process. The amplitude of the signal $S$ has a constant offset of $A_{\text {Sig }}^{2}+A_{\text {Ref }}^{2}$ and is modulated by $v_{\mathrm{d}}$, the frequency of the

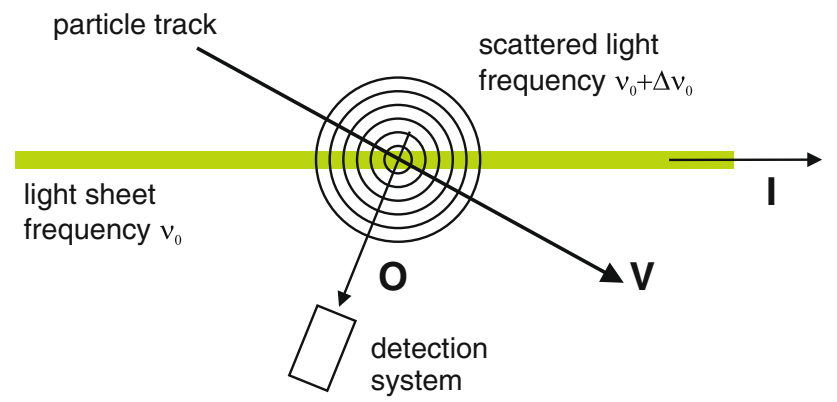

Fig. 1 Geometry of the DGV setup
Doppler shift. The offset is usually large compared to the amplitude of the modulated part of the signal as $A_{\mathrm{Sig}}$ is much smaller than $A_{\text {Ref. }}$ In general, there will be more than one particle in the measurement volume scattering the light. Each of the particles will create a light wave with a random phase. The interference of these light waves with the reference beam creates a signal with the same Doppler shift but with a reduced modulation depth.

\subsection{Detection and signal processing}

Using standard camera technology the signal described in Eq. 2 is not detectable. Even for low velocities the frequencies are too high and the modulation depth is too low. One way to detect such signals would be the use of multiple anode photomultiplier arrays. These devices offer the ability to demodulate frequencies up to about $1 \mathrm{GHz}$. But each of the pixels of these arrays requires its own data aqcuisition chain resulting in a fairly complex system for the rather low resolution it offers. Another possibility is the use of smart pixel detector arrays. Those detector arrays offer at the pixel level not only the ability to detect light but also some basic pre-processing of the signal. Recent advances in parallel Optical Coherence Tomography have led to the development of the pOCTii detector by Beer et al. (2005) which is now produced by the company Heliotis. This smart pixel detector array offers a resolution of $144 \times 90$ pixels and performs a dual phase lock-in detection on each pixel with a maximum demodulation frequency of $50 \mathrm{kHz}$. It additionally offers a background compensation on each pixel increasing its sensitivity up to $86 \mathrm{~dB}$. This background compensation effectively removes the constant offset in the signal $S$ (Eq. 2).

Dual phase lock-in detectors are commonly used to extract the amplitude and phase of weak periodic signals of known frequency from a noisy background. They are based on the mutual ortohogonality property of sinusoidal functions described in Eqs. 3 and 4:

$$
\begin{aligned}
I & =\left\langle A \cos \left(2 \pi v_{\mathrm{d}} t+\phi\right) \cos \left(2 \pi v_{\mathrm{D}} t\right)\right\rangle \\
& =\left\{\begin{array}{c}
\frac{A}{2} \cos (\phi) \text { if } v_{\mathrm{D}}=v_{\mathrm{d}} \\
0 \text { otherwise }
\end{array}\right. \\
Q & =\left\langle A \cos \left(2 \pi v_{\mathrm{d}} t+\phi\right) \sin \left(2 \pi v_{\mathrm{D}} t\right)\right\rangle \\
& =\left\{\begin{array}{c}
\frac{A}{2} \sin (\phi) \text { if } v_{\mathrm{D}}=v_{\mathrm{d}} \\
0 \text { otherwise }
\end{array}\right.
\end{aligned}
$$

The periodic input signal with the frequency $v_{\mathrm{d}}$ and phase $\phi$ is multiplied with a periodic reference signal at the demodulation frequency $v_{\mathrm{D}}$ and averaged over a sufficiently long time. The output of the lock-in detector is only non-zero if the signal frequency $v_{\mathrm{d}}$ and the demodulation frequency $v_{\mathrm{D}}$ are the same. The resulting in-phase $(I)$ and quadrature $(Q)$ components can be used to 
calculate the amplitude $A$ and the phase $\phi$ of the signal (Eqs. 5, 6).

$A=\sqrt{I^{2}+Q^{2}}$

$\phi=\arctan \left(\frac{Q}{I}\right)$

This lock-in detection is implemented for each pixel on the detector as follows: The signal is first integrated and sampled at four times the demodulation frequency $v_{\mathrm{D}}$. These samples are then multiplied by a periodic signal with frequency $v_{\mathrm{D}}$ and the discrete values $[1,0,-1,0]$ for the $I$ component and $[0,1,0,-1]$ for the $Q$ component. These discrete values replace the sine and the cosine of the lock-in detection algorithm in Eqs. 3 and 4. The signals are then averaged and read out. Eqs. 7 and 8 describe this process:

$I=\sum_{i=0}^{N-1}\left(\int_{4 i T_{\mathrm{qp}}}^{(4 i+1) T_{\mathrm{qp}}} S(t) \mathrm{d} t-\int_{(4 i+2) T_{\mathrm{qp}}}^{(4 i+3) T_{\mathrm{qp}}} S(t) \mathrm{d} t\right)$

$Q=\sum_{i=0}^{N-1}\left(\int_{(4 i+1) T_{\mathrm{qp}}}^{(4 i+2) T_{\mathrm{qp}}} S(t) \mathrm{d} t-\int_{(4 i+3) T_{\mathrm{qp}}}^{(4 i+4) T_{\mathrm{qp}}} S(t) \mathrm{d} t\right)$

$S(t)$ is the signal to be detected, $T_{\mathrm{qp}}$ denotes the integration time and $N$ is the number of periods to be averaged.

The averaging process cannot be performed over infinitely long times. Figures 2 and 3 show the effects of the finite averaging intervals for two different averaging lengths $N$ and three different phases $\phi$ on the calculated amplitude $A$ as a function of $v_{\mathrm{d}}$ for a demodulation frequency $v_{\mathrm{D}}$ of $50 \mathrm{kHz}$. Figure 2 shows the result for averaging over $N=10$ periods of the reference signal, Fig. 3 the result for averaging over $N=1$ period. The peak width increases and the influence of the phase offset $\phi$ grows with decreasing averaging length. In the extreme case of averaging only over one period a signal is obtained over the frequency range from $0 \mathrm{~Hz}$ up to twice the demodulation frequency.

The use of the detector as a lock-in detector would be the direct approach to detect the Doppler shift. But for a flow measurement this approach does not offer satisfying performance: For every possible Doppler shift present in the scene the lock-in detection has to be performed for at least $N=20$ periods to receive a reasonably accurate result. This leads to long acquisition times as a complete frequency sweep has to be performed.

Another more promising approach, which is widely used in Doppler radar systems, is based on the pulse pair statistics method described by Miller et al. (1972). This method relies on taking the derivative of the measured phase, since the deviation of the signal frequency from the

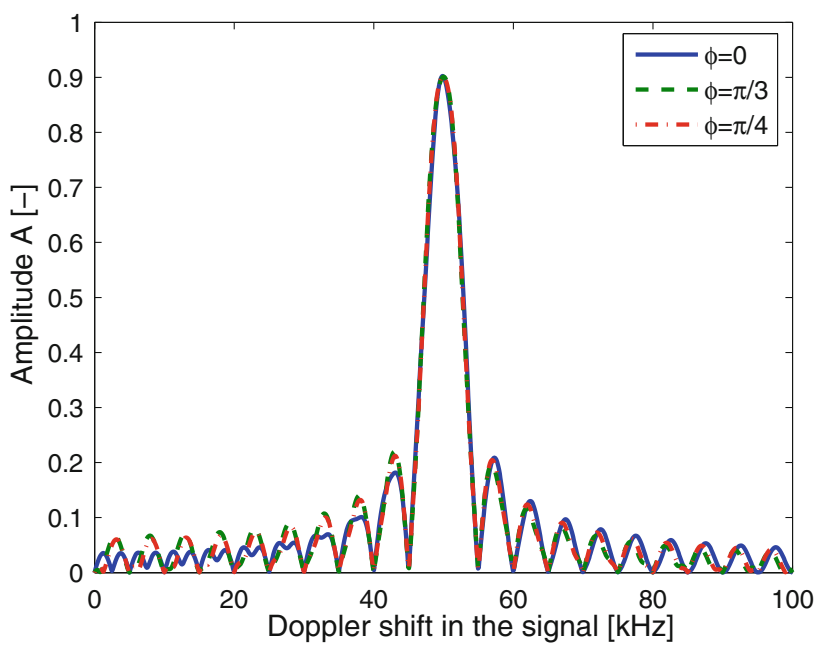

Fig. 2 Calculated output amplitude of the lock-in detection as a function of the input signal averaged over $N=10$ periods for three different phase shifts

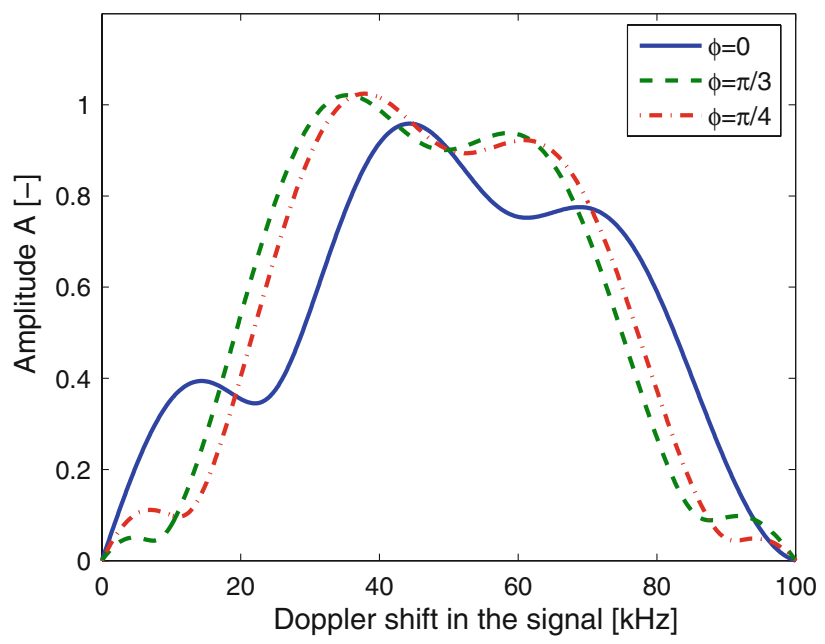

Fig. 3 Calculated output amplitude of the lock-in detection as a function of the input signal averaged over $N=1$ periods for three different phase shifts

demodulation frequency can also be seen as a time-varying phase:

$S=A \sin \left(2 \pi v_{\mathrm{d}} t+\phi\right) \equiv A \sin \left(2 \pi v_{\mathrm{D}} t+\varphi(t)\right)$

with $\varphi(t)=\phi+\left(v_{\mathrm{d}}-v_{\mathrm{D}}\right) t$

For the computation of pulse pair statistics the $I$ and the $Q$ components are combined into a complex signal $C(t)$ and its autocorrelation $R(\tau)$ is calculated:

$C(t)=I(t)+i Q(t)$

$R(\tau)=C(t) C^{*}(t+\tau)$

Based on $R(\tau)$ the frequency of the detected signal can be estimated: 
$\hat{v}_{d}=\frac{\arctan (\Im\{R(\tau)\} / \Re\{R(\tau)\})}{2 \pi \tau}$

Combining the Eqs. 7, 8 and 12 yields:

$$
\begin{gathered}
\hat{v}_{\mathrm{d}}=\frac{-1}{2 \pi \tau} \arctan \left(\frac{2 \sin \left(2 \pi v_{\mathrm{d}} \tau\right) \sin \left(2 \pi v_{\mathrm{d}} T_{\mathrm{qp}}\right)}{E+F+G}\right), \\
\text { with } E=2 \cos \left(2 \pi v_{\mathrm{d}} \tau\right) \\
\text { and } F=\cos \left(2 \phi+2 \pi v_{\mathrm{d}}\left(\tau-T_{\mathrm{qp}}+4 N T_{\mathrm{qp}}\right)\right) \\
\text { and } G=\cos \left(2 \phi+2 \pi v_{\mathrm{d}}\left(\tau+T_{\mathrm{qp}}+4 N T_{\mathrm{qp}}\right)\right)
\end{gathered}
$$

The right hand side of Eq. 13 still depends on the random phase $\phi$ in the signal $S$ (Eq. 2). Averaging $R(\tau)$ over several measurements eliminates this dependency:

$\hat{v}_{\mathrm{d}}=\frac{-1}{2 \pi \tau} \arctan \left(\tan \left(2 \pi v_{\mathrm{d}} \tau\right) \sin \left(2 \pi v_{\mathrm{d}} T_{\mathrm{qp}}\right)\right)$.

Figure 4 shows $\hat{v}_{\mathrm{d}}\left(v_{\mathrm{d}}\right)$ for $\tau=T_{\mathrm{qp}}$. The shift estimate is not a linear function of the frequency and thus the frequency estimation is based on Eq. 14 is not accurate. Since $\hat{v}_{\mathrm{d}}\left(v_{\mathrm{d}}\right)$ is injective the results can be inverted numerically and $v_{\mathrm{d}}$ can be determined uniquely using the estimate $\hat{v}_{\mathrm{d}}$. Additionally, this estimator allows the demodulation of frequencies up to twice $v_{\mathrm{D}}$. Equation 14 assumes that there is only one particle in the measurement volume. For the general case with several particles scattering the light the signal $S$ will have a ceratin bandwidth. In the case of a symmetrical bandwidth the calculated velocity based on $\hat{v}_{\mathrm{d}}$ is the mean velocity in the probe volume. In the case of an asymmetrical bandwidth the calculated velocity is biased. But this bias is usually small (Sirmans et al. 1975).

The ideal HDGV setup uses two pOCTii detectors operating in parallel with the same demodulation frequency and lock-in averaging time $N$, but with an independantly controlled time delay $\tau$ between the two detectors. To maximize the measurable frequency range $N$ should be

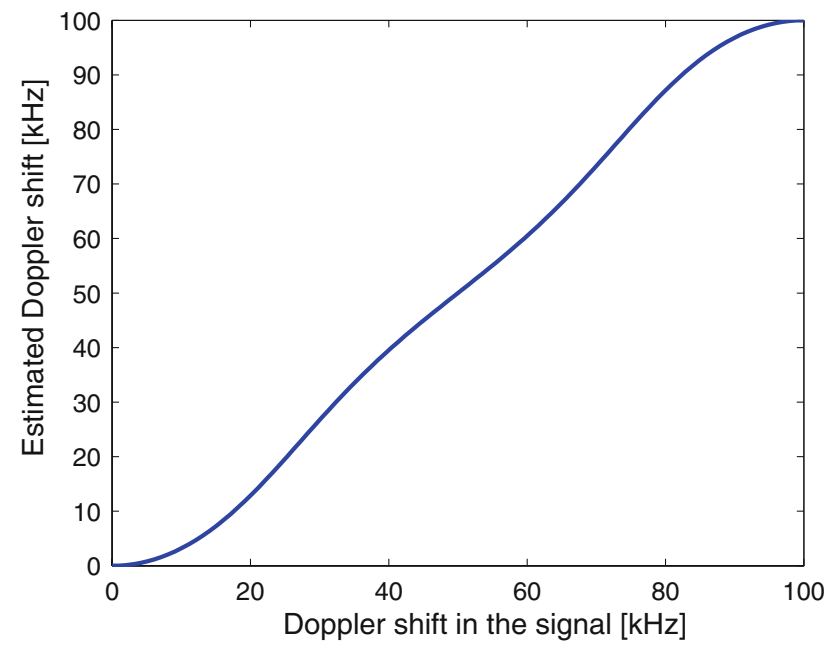

Fig. 4 Estimated frequency shift as a function of the real frequency shift for $\tau=T_{\mathrm{qp}}$ chosen to be 1 and $\tau$ should be equal to $T_{\mathrm{qp}}$. The presented experiments were performed using only one detector, thus the autocorrelation $R(\tau)$ is calculated using two subsequently acquired I-Q pairs with a time delay $\tau=16 T_{\mathrm{qp}}$ between the two images for the first experiment and $\tau=6 T_{\mathrm{qp}}$ for the second experiment. Figure 5 shows the impact of this increased time delay onto the Doppler estimator. An additional wrapping of the frequency estimates results. The phase unwrapping problem can be solved in a least-squares sense using for example the fast cosine transforms as suggested by Ghiglia et al. (1994).

\section{Experiments}

Two experiments are presented in this section. The first compares the measured velocity distribution of a rotating disk with its true velocity. This experiment is well suited to validate the measurement technique. The second experiment shows the measurement of the velocity distribution of a buoyant plume above a heated resistor, validating the method in a real flow. Figure 6 shows a typical HDGV set up as it was used for the experiments. The source laser beam of a CW laser (Coherent Verdi V5, $\lambda_{0}=532 \mathrm{~nm}$, max. power $5 \mathrm{~W}$ ) is split into two parts using a Fresnel beam sampler. One beam containing $95 \%$ of the optical power is coupled into a fiber optic delivery system. Different front-ends can be attached depending on the illumination requirements. The reference beam containing the remaining $5 \%$ of the power is redirected towards the camera using several mirrors. Figure 7 shows a close-up of the camera. The camera lens projects the scene onto the pOCTii detector while a separate two-lens system is used to expand the reference beam to cover the same detector

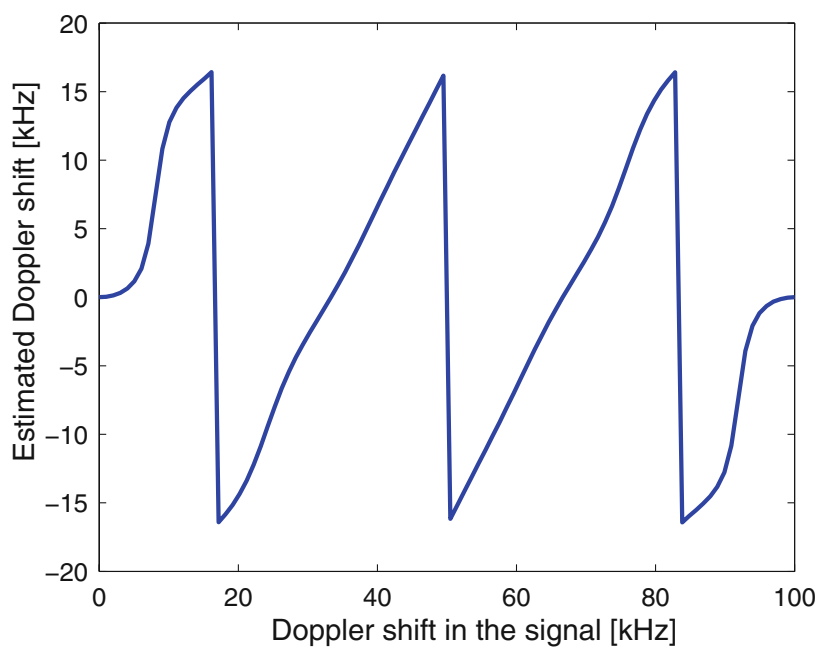

Fig. 5 Estimated frequency shift as a function of the real frequency shift for $\tau=6 T_{\mathrm{qp}}$ 


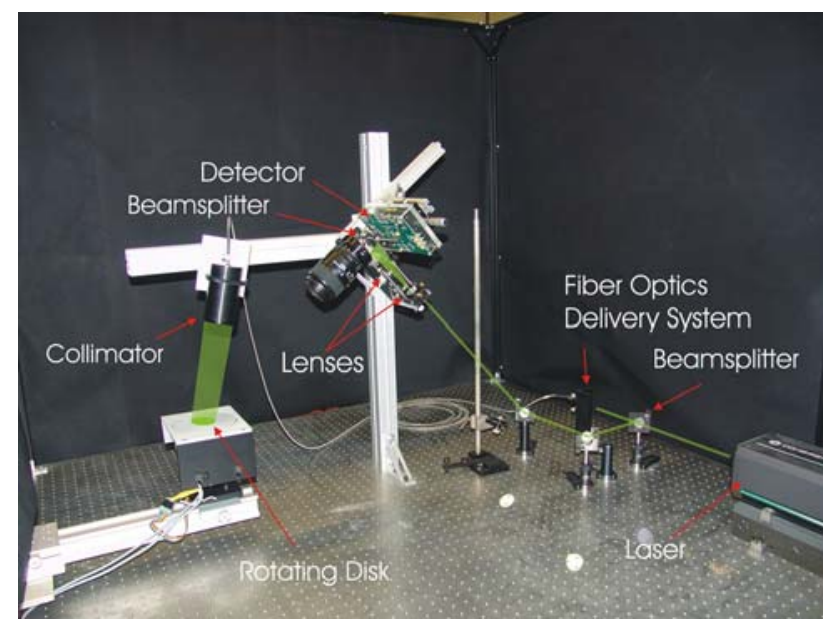

Fig. 6 Setup of the HDGV measurement system

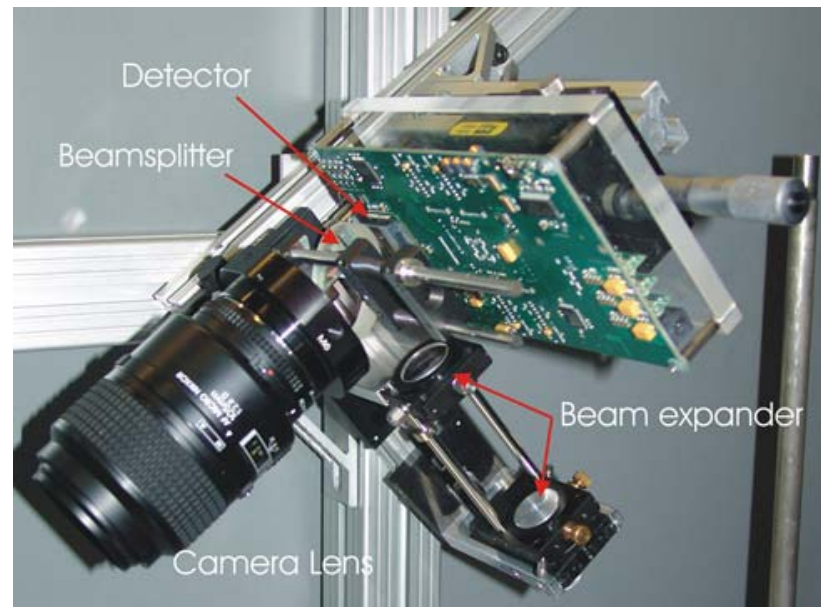

Fig. 7 Close up of the Doppler camera system

area. The beam splitter is used to overlay the light from the scene and the light from the reference beam resulting in an effective reference beam power of approx. $1-2 \%$.

\subsection{Rotating disk experiment}

The setup shown in Fig. 6 was used to measure the (known) velocity of a rotating disk. To illuminate the disk a collimator front-end was attached to the fiber optic delivery system. The resulting expanded laser beam had a diameter of $50 \mathrm{~mm}$. The power of the laser was set to $0.5 \mathrm{~W}$. The measured area on the rotating disk was roughly $20 \mathrm{~mm} \times 40 \mathrm{~mm}$. The rotational frequency of the disk was set to $0.73 \mathrm{~Hz}$ and the demodulation frequency $v_{\mathrm{D}}$ was set to $13.02 \mathrm{kHz}$ averaging over $N=1$ periods of the lock-in process. For the correlation averaging 300 images were recorded.

Figure 8 at the top presents the resulting velocity map. It shows the expected linear distribution: The projected velocity is constant on straight lines and increases linearly towards the outside of the disk. It also shows the directional ambiguity of the velocity measurement. Figure 8 at the bottom shows the standard deviation of the measurement calculated along the direction with constant velocity. Except for the very low and high velocities the standard deviation is lower than $0.25 \mathrm{~mm} / \mathrm{s}$. This corresponds to a standard deviation of $250 \mathrm{~Hz}$ for the frequency estimation or less than $1 \%$ of the maximum detectable frequency. This increasing standard deviation towards the lower and upper end of the measurement range is related to the detected amplitude shown in Fig. 3. The I-Q demodulation of the detector acts as a band-pass filter even when the signal is averaged only over $N=1$ cycle. High and low frequencies are damped and the signal-to-noise ratio drops. Figure 9 compares the true velocity of the rotating disk along a line with the measured velocity. At the top, Fig. 9 shows the measured velocities and the true velocity of the disk and the bottom shows the error of the measurement. Although all the calibration parameters of the system were only measured and not calibrated by an independant measurement the measurement shows a good agreement. The calculated error indicates that the slope is matched very well but there is a bias of $-0.5 \mathrm{~mm} / \mathrm{s}$. The source of this small bias is yet unclear but as it is constant over the whole frequency range a calibration measurement would remove it.

\subsection{Buoyant plume experiment}

Figure 10 shows a picture of the setup as used to measure the velocity above a heated resistor. The resistor is placed in a plexiglas tube which is closed at the top and the bottom, and the flow was seeded using smoke particles from joss sticks. For this experiment the scene was illuminated with a planar laser sheet. The geometrical arrangement of the observation and illumination directions was chosen such that the detected Doppler shift corresponds to the vertical velocity component of the flow. The output power of the laser was increased to $4 \mathrm{~W}$ and the demodulation frequency $v_{\mathrm{D}}$ was set to $4.35 \mathrm{kHz}$, performing the lock-in detection over $N=1$ periods. For the averaging 300 images were recorded.

Figure 11 shows the resulting velocity map. The shape of the plume is clearly visible. Due to the asymmetrical setup and the elongated shape of the heat source the measured flow distribution is not symmetrical either. The directional ambiguity of the technique can be noticed again in the right part of the image. The error and standard deviation measured in the rotating disk experiment cannot be directly used to asses the accuracy of the plume measurement since a different demodulation frequency and a different geometry were employed. Nevertheless the low standard deviation seen in Sect. 3.1 is also visible in Fig. 11 as the surface is quite smooth. 
Fig. 8 Velocity map of the measured projected velocity of the rotating disk (top). Standard deviation of the measurement (bottom)
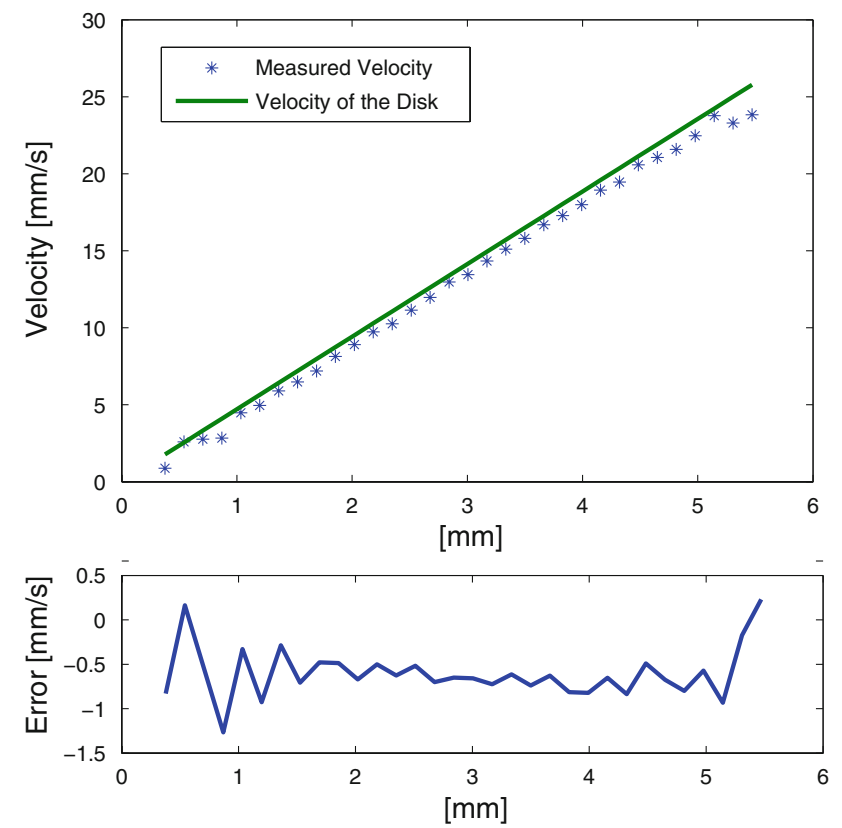

Fig. 9 Comparison of the measured and the true velocity of the rotating disk (top). Error of the velocity measurement (bottom)

\section{Discussion and outlook}

The two experiments demonstrate the basic feasibility of the direct demodulation/heterodyning concept using a smart pixel detector array. The signal processing based on

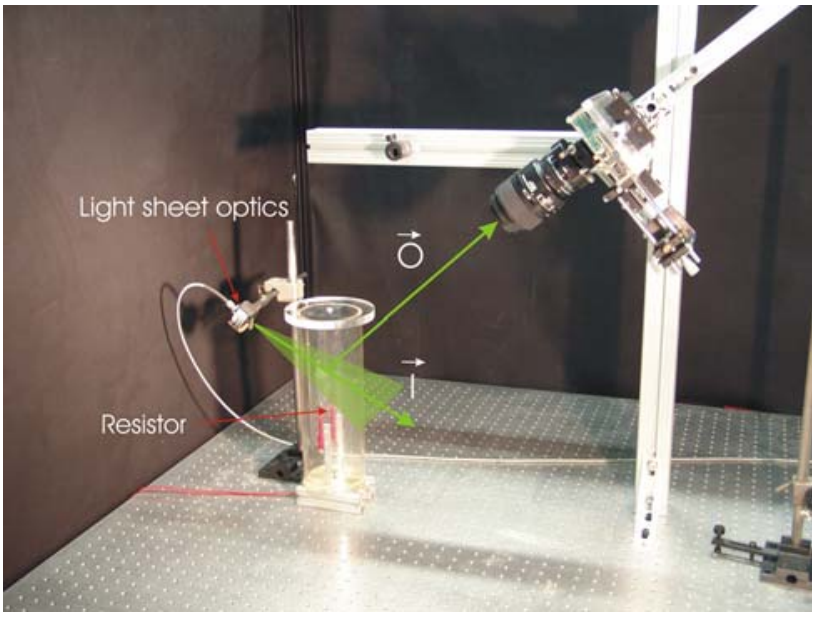

Fig. 10 Setup of the buoyant plume experiment

pulse-pair statistics offers the possibility to detect complete frequency shift distributions without the necessity for a frequency sweep of the detection device.

The current sensor array has demonstrated detection capabilities in a real flow extending to Doppler frequencies of about $4.5 \mathrm{kHz}$. This corresponds, depending on the viewing geometry and the dominant flow direction, to a detectable velocity range of about $3 \mathrm{~mm} / \mathrm{s}$. This is sufficient already for a number of microfluidic applications. For higher flow velocities the available power of the laser was too low. The rotating disk experiment demonstrates that 
Fig. 11 Velocity map of the measured projected velocity of the buoyant plume

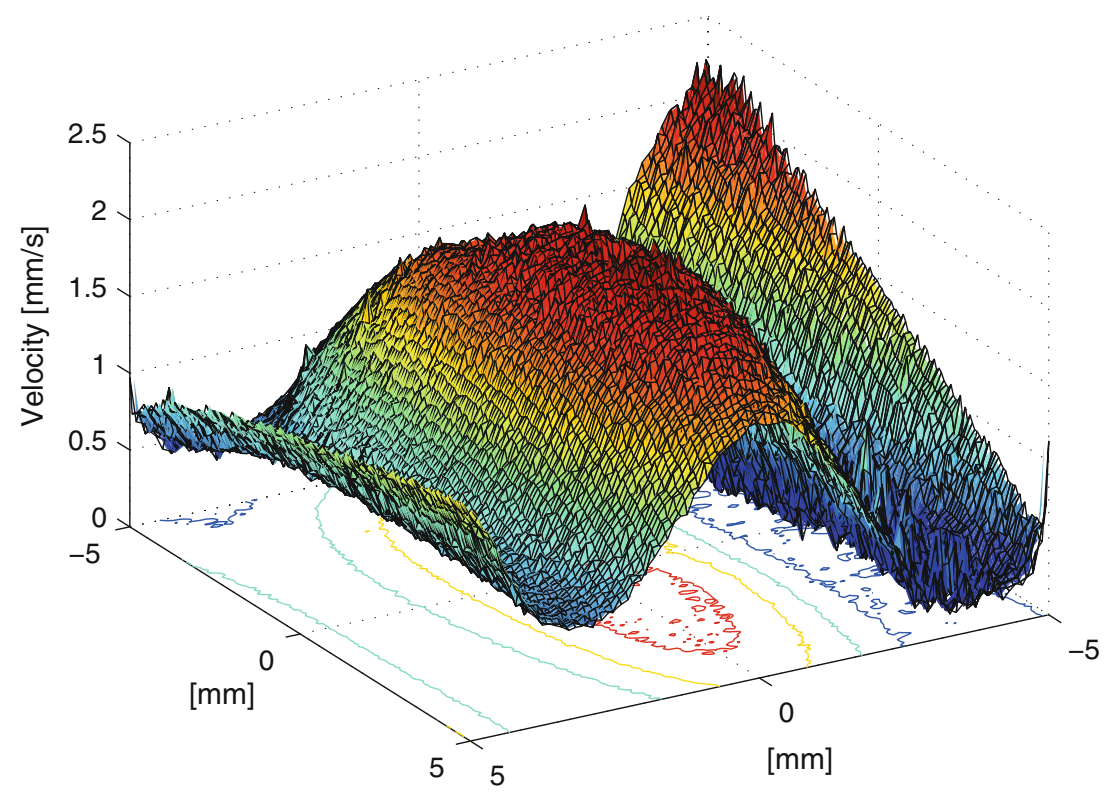

with sufficient power Doppler frequencies up to $26 \mathrm{kHz}$ can be detected.

Recent improvements in the firmware of the detector will allow the demodulation of Doppler shifts up to $500 \mathrm{kHz}$. For the geometrical arrangement of the illumination and observation direction as it was used in the present experiments, those Doppler shifts correspond to a velocity of approximately $0.2 \mathrm{~m} / \mathrm{s}$. The velocity range may be further extended using a frequency modulated reference beam, shifting higher Doppler frequencies down to the demodulation frequency range of the detector. Using a premodulated reference beam also eliminates the directional ambiguity and the problem with the increased standard deviation for velocities close to $0 \mathrm{~mm} / \mathrm{s}$. Similar to the classical dual beam laser Doppler arrangement for point measurements (vom Stein et al. 1969), a crossed lightsheet geometry as presented by Hachiga et al. (1998) or a crossed light-sheet geometry with co-planar light sheets may also be advantageous for HDGV. Such systems offer several advantages compared to the present reference beam set up. The optical mixing is performed in the flow with two different illumination directions and no longer on the detector using a reference beam. The measured Doppler shifts no longer depend on the geometrical orientation of $\mathbf{O}$ and I but only on the two illumination directions. Thus the measurement range and the measured velocity component are no longer limited by the viewing angle of the camera. With the two different crossed light sheet configurations either the out-of-plane velocity or one component of the inplane velocity are measurable and, using very small crossing angles of $1^{\circ}$ or less, the velocity range will be increased to approx. $10 \mathrm{~m} / \mathrm{s}$. Nevertheless those systems have a major drawback: The power requirement on the light source is increased as the reference beam can no longer be used to amplify the weak modulation in the signal. Such systems are currently being evaluated and will be subject to an upcoming publication.

\section{References}

Ainsworth RW, Thorpe SJ, Manners RJ (1997) A new approach to flow-field measurement-a view of Doppler global velocimetry techniques. Int J Heat Fluid Flow 18:116-130

Beer S, Seitz P (2005) Real-time tomographic imaging without $\mathrm{X}$-rays: a smart pixel array with massively parallel signal processing for real-time optical coherence tomography performing close to the physical limits. Research in Microelectronics and Electronics $2005 \mathrm{PhD}$ 2:135-138

Beutner TJ, Elliott GS, Mosedale A, Carter C (1998) Doppler global velocimetry applications in large scale facilities. In: AIAA 20th advanced measurement and ground testing conference, AIAA Paper 98-2608

Elliott GS, Beutner TJ (1999) Molecular filter based planar Doppler velocimetry. Prog Aerosp Sci35:799-845

Ghiglia DC, Romero LA (1994) Robust two-dimensional weighted and unweighted phase unwrapping that uses fast transforms and iterative methods. J Opt Soc Am A11:107-117

Hachiga T, Furuichi N, Mimatsu J, Hishida K, Kumada M (1998) Development of a multi-point LDV by using semiconductor laser with FFT-based multi-channel signal processing. Exp Fluids 24:70-76

Miller KS, Rochwarger MM (1972) A covariance approach to spectral moment estimation. IEEE Trans Info Theory 18: 588-596

Roehle I, Schodl R, Voigt P and Willert C (2000) Recent developments and applications of quantitative laser light sheet measuring techniques in turbomachinery components. Meas Sci Technol 11:1023-1035 
Samimy M, Wernet MP (2000) Review of planar multiplecomponent velocimetry in high-speed flows. AIAA J 38:553574

Sirmans D, Bumgarner B (1975) Numerical comparison of five mean frequency estimators. J Appl Meteor 14:991-1003
Smith MW, Drummond JP, Northam GB (1996) Application of absorption filter planar Doppler velocimetry to sonic and supersonic jets. AIAA J 34:434-441

vom Stein HD, Pfeiffer HJ (1969) A Doppler difference method for velocity measurements. Metrologia 5:59-61 